\title{
COMPENSADORES DINÂMICOS PARA SISTEMAS DISCRETOS NO TEMPO COM PARÂMETROS VARIANTES E APLICAÇÃO A UM SISTEMA FUZZY TAKAGI-SUGENO
}

\author{
Michael Klug* \\ micklug@das.ufsc.br
}

\author{
Eugênio B. Castelan* \\ eugenio@das.ufsc.br
}

${ }^{*}$ Grupo de Controle de Sistemas Mecatrônicos - CSM

Depto. de Automação e Sistemas - DAS

Universidade Federal de Santa Catarina - UFSC

Florianópolis, 88040-900, SC, Brasil

\begin{abstract}
Dynamic Compensators for Discrete-Time Systems with Varying Parameters and Application to a Takagi-Sugeno Fuzzy System

In this article we consider the synthesis of dynamic output feedback compensators for linear discrete-time parameter-varying systems. By assuming that the timevarying parameters can be measured or estimated in real-time, two full-order compensators are considered: one that is partially dependent on the parameters and other that is totally dependent. Closed-loop stability and time performance are verified via a parameter dependent Lyapunov function. LMI conditions are given for the synthesis of the controllers. To cope with practical applications, particularly in the case where the timevarying linear model represents locally the dynamics of a nonlinear plant, additional conditions are also considered to take into account the local validity of the model. The proposed results are applied to a nonlinear system that is locally represented by a Takagi-Sugeno fuzzy model.
\end{abstract}

KEYWORDS: Dynamic compensators, LPV systems, Parameter dependent control, Takagi-Sugeno fuzzy models, LMIs.

\footnotetext{
Artigo submetido em 16/06/2011 (Id.: 1346)

Revisado em 25/09/2011, 01/12/2011

Aceito sob recomendação do Editor Associado Prof. Luis Fernando Alves
} Pereira

\section{RESUMO}

Neste artigo, considera-se o problema de síntese de compensadores dinâmicos por realimentação de saídas para sistemas lineares, discretos no tempo, com parâmetros variantes. Sob a hipótese que os parâmetros variantes podem ser medidos ou estimados em tempo-real, são consideradas duas estruturas de compensadores dinâmicos, com ordem igual à da planta: uma parcialmente dependente e outra completamente dependente dos parâmetros. A estabilidade e o desempenho do sistema em malha-fechada são garantidos via a utilização de uma função de Lyapunov dependente de parâmetros e condições LMIs são estabelecidas e utilizadas para a síntese dos controladores. Um aspecto importante considerado, e que objetiva a aplicação prática dos resultados, particularmente no controle de sistemas não lineares, é a validade do modelo LPV utilizado para representar localmente o sistema a controlar. Os resultados propostos são aplicados sobre um sistema não linear representado localmente por um modelo fuzzy de Takagi-Sugeno.

PALAVRAS-ChaVE: Compensadores dinâmicos, Sistemas LPV, Controle dependente de parâmetros, Modelos fuzzy Takagi-Sugeno, LMIs. 


\section{INTRODUÇÃO}

Sistemas lineares com parâmetros variantes (ou, sistemas LPV) têm recebido atenção especial da comunidade científica, particularmente na área de controle, pois permitem, por exemplo, modelar ou aproximar o comportamento dinâmico de sistemas não lineares entre diferentes pontos de funcionamento ou numa dada região do espaço de estados. Dentre as formas de representação de sistemas LPV, a modelagem na forma politópica tem sido largamente explorada na literatura, com contribuições interessantes de vários pesquisadores brasileiros com colaboradores, reportadas em revistas e congressos nacionais (p.ex.: Oliveira et al., 2002; Leite et al., 2004; Araújo et al., 2010) e internacionais (p.ex.: Trofino and Souza, 2001; Geromel and Colaneri, 2006; Montagner et al., 2005; Souza and Trofino, 2005; Oliveira and Peres, 2008; Mozelli and Palhares, 2011); veja também a bibliografia mencionada nestas referências.

Na representação politópica, as matrizes do sistema pertencem à um politopo de matrizes, definido por seus vértices e por um vetor de parâmetros dependente do tempo e pertencente ao simplex unitário. Caso este vetor de parâmetros possa ser medido ou seus elementos possam ser estimados em tempo real, pode-se buscar o atendimento das especificações desejadas para o sistema em malha fechada via a utilização de uma lei de controle dependente dos parâmetros. Para a síntese dessas leis de controle variantes no tempo, técnicas baseadas na utilização de Funções de Lyapunov Dependentes de Parâmetros e em formulações via Desigualdades Matriciais Lineares têm sido exploradas na busca de soluções menos conservadoras para os diversos problemas relacionados. Do ponto de vista prático, diversas aplicações têm sido reportadas na literatura e, em particular, a utilização da modelagem fuzzy de Takagi-Sugeno (Wang et al., 1996) apresenta-se como uma área interessante para a aplicação de técnicas de controle LPV e, também, para o desenvolvimento de novos resultados teóricos (p.ex.: Guerra and Vermeiren, 2004; Khiar et al., 2007; Andrea et al., 2008; Tognetti et al., 2009; Montagner et al., 2010; Zheng et al., 2001; Zhou et al., 2007).

Na representação fuzzy de Takagi-Sugeno (T-S) o parâmetro variante consiste em funções peso normalizadas, geralmente dependente dos estados do sistema, que controlam a interpolação entre os modelos locais (regras). Estas regras podem então ser calculadas e utilizadas para o controle digital de sistemas não lineares, via o modelo T-S. Uma outra característica importante é que o modelo obtido representa exatamente, ou aproxima, o sistema não linear original somente na região do espaço de estados considerada para a obtenção do modelo T-S. Esta característica, importante do ponto de vista da aplicação prática de controladores, e que também pode estar presente quando se modela o sistema a controlar por outras técnicas para obtenção de modelos $\mathrm{LPV}$, é geralmente desconsiderada na fase de projeto, quando condições de estabilidade ou desempenho globais são usadas para a síntese do controlador.

Dentro do contexto brevemente descrito anteriormente, os objetivos principais deste artigo são:

1. Apresentar duas estruturas de compensadores dinâmicos por realimentação de saída para sistemas LPV, com dependência parcial e dependência total dos parâmetros variantes, especializando os resultados propostos em (Castelan et al., 2010; Klug et al., 2011) para o caso de sistemas LPV considerados no presente trabalho;

2. Levar em consideração a região de validade do modelo na fase de projeto dos compensadores: o objetivo, neste caso, será determinar uma região de condições iniciais, $S_{0}$, contida no domínio de validade e tão grande quanto possível, com a garantia que as trajetórias que iniciam em $S_{0}$ evoluam no interior do domínio de validade do modelo LPV considerado;

3. Aplicar e comparar as técnicas desenvolvidas em um determinado modelo fuzzy T-S.

O artigo é organizado da forma seguinte: na Seção 2 é apresentado o sistema LPV considerado e o problema de controle a ser desenvolvido; na Seção 3 mostram-se alguns resultados preliminares e definições utilizadas neste trabalho; na seção 4 são deduzidas as condições de síntese dos controladores dinâmicos, são abordadas as condições adicionais para modelos locais e formulados os problemas de programação convexa utilizados para a síntese dos controladores; um exemplo numérico e comentários são apresentados na Seção 5; por fim apresentam-se as conclusões obtidas.

Notacões. Para um vetor $x \in \Re^{n}, x \geq 0$ significa que todos os componentes de $x$, denotados por $x_{(i)}$, são não negativos. Os elementos de uma matriz $A \in \Re^{m \times n}$ são denotados por $A_{(i, j)}, i=1, \ldots, m, j=1, \ldots, n . A_{(i)}$ denota a iésima linha da matriz $A$. Para duas matrizes simétricas, $A$ e $B, A>B$ significa que $A-B$ é positiva definida. $A^{\prime}$ denota a transposta de $A$. $I_{m}$ denota a matriz identidade de ordem $m$ e $\operatorname{diag}(x)$ denota a matriz diagonal obtida do vetor $x$. O símbolo $*$ representa blocos simétricos e $\bullet$ representa um elemento que não possui influência no desenvolvimento. 


\section{APRESENTAÇÃO DO PROBLEMA}

Considere um sistema linear em tempo discreto com parâmetros variantes no tempo, representado por:

$$
\left\{\begin{array}{l}
x_{k+1}=A\left(h_{k}\right) x_{k}+B\left(h_{k}\right) u_{k} \\
y_{k}=C x_{k}
\end{array}\right.
$$

em que $x_{k} \in \Re^{n}, u_{k} \in \Re^{m}$ e $y_{k} \in \Re^{p}$ são, respectivamente, os vetores dos estados, das entradas de controle e das saídas do sistema. Além disso, $h_{k} \subset \Re^{r}$ é um vetor de parâmetros, variante no tempo, mensurável ou possível de ser estimado em tempo real, e limitado no simplex unitário $\Xi=\left\{h_{k} \in \Re^{r} ; \sum_{i=1}^{r} h_{k(i)}=1, h_{k(i)} \geq\right.$ $0, i=1, \ldots r\}$.

A estrutura das matrizes do sistema possui a forma seguinte:

$$
\left[\begin{array}{ll}
A\left(h_{k}\right) & B\left(h_{k}\right)
\end{array}\right]=\sum_{i=1}^{r} h_{k(i)}\left[\begin{array}{ll}
A_{i} & B_{i}
\end{array}\right]
$$

e $C \in \Re^{p \times n}$, com $A_{i} \in \Re^{n \times n}$ e $B_{i} \in \Re^{n \times m}$.

O problema de controle a ser investigado consiste da síntese de um controlador dinâmico por realimentação de saída (CDRS) que garanta a estabilidade para o sistema correspondente em malha fechada, obedecendo um certo requisito de desempenho a ser definido posteriormente.

Para o CDRS, admite-se dois casos:

\section{CASO 1 - CDRS Parcialmente Dependente de Parâmetros}

Seja o controlador dinâmico

$$
\begin{cases}x_{c, k+1} & =A_{c}\left(h_{k}\right) x_{c, k}+B_{c}\left(h_{k}\right) u_{c, k} \\ y_{c, k} & =C_{c} x_{c, k}+D_{c} u_{c, k}\end{cases}
$$

em que $x_{c} \in \Re^{n}, y_{c, k} \in \Re^{m}$ e $u_{c, k} \in \Re^{p}$. Considera-se a seguinte estrutura das matrizes do controlador

$$
\begin{aligned}
& {\left[\begin{array}{cc}
A_{c}\left(h_{k}\right) & B_{c}\left(h_{k}\right)
\end{array}\right]=} \\
& \sum_{i=1}^{r} h_{k(i)}\left[\begin{array}{ll}
A_{c i} & B_{c i}
\end{array}\right]
\end{aligned}
$$

e $C_{c} \in \Re^{m \times n}, D_{c} \in \Re^{m \times p}$, na qual $A_{c i} \in \Re^{n \times n}$ e $B_{c i} \in$ $\Re^{n \times p}$. Como as matrizes da equação de saída, $C_{c}$ e $D_{c}$, não dependem do parâmetro variante, nomeia-se este controlador de CDRS-PDP (Parcialmente Dependente de Parâmetros).

Considerando a interconexão $u_{c, k}=y_{k}$ e $y_{c, k}=u_{k}$, temse o seguinte sistema em malha fechada:

$$
\left\{\begin{aligned}
x_{k+1}= & A\left(h_{k}\right) x_{k}+B\left(h_{k}\right) C_{c} x_{c, k}+ \\
& B\left(h_{k}\right) D_{c} C x_{k} \\
x_{c, k+1}= & A_{c}\left(h_{k}\right) x_{c, k}+B_{c}\left(h_{k}\right) C x_{k}
\end{aligned}\right.
$$

Então, definindo o vetor de estados aumentado $\varsigma_{k}=$ $\left[\begin{array}{ll}x_{k}^{\prime} & x_{c, k}^{\prime}\end{array}\right]^{\prime}$, o sistema em malha fechada pode ser representado por:

$$
\varsigma_{k+1}=\left(\mathbb{A}\left(h_{k}\right)+\mathbb{B}\left(h_{k}\right) \mathbb{K}\right) \varsigma_{k}
$$

com

$$
\begin{gathered}
\mathbb{A}\left(h_{k}\right)=\left[\begin{array}{cc}
A\left(h_{k}\right) & 0 \\
B_{c}\left(h_{k}\right) C & A_{c}\left(h_{k}\right)
\end{array}\right], \quad \mathbb{B}\left(h_{k}\right)=\left[\begin{array}{c}
B\left(h_{k}\right) \\
0
\end{array}\right] \\
e \mathbb{K}=\left[\begin{array}{ll}
D_{c} C & C_{c}
\end{array}\right] .
\end{gathered}
$$

Observa-se de (5) que a matriz $\mathbb{K}$ pode ser vista como uma realimentação constante dos estados do sistema aumentado.

\section{CASO 2 - CDRS Totalmente Dependente de Parâmetros}

Seja o controlador dinâmico

$$
\left\{\begin{array}{l}
x_{c, k+1}=A_{c}\left(h_{k}\right) x_{c, k}+B_{c}\left(h_{k}\right) u_{c, k} \\
y_{c, k}=C_{c}\left(h_{k}\right) x_{c, k}+D_{c}\left(h_{k}\right) u_{c, k}
\end{array}\right.
$$

com a seguinte estrutura das matrizes do controlador:

$$
\begin{gathered}
{\left[\begin{array}{cc}
A_{c}\left(h_{k}\right) & B_{c}\left(h_{k}\right)
\end{array}\right]=\sum_{i=1}^{r} h_{k(i)}^{2}\left[\begin{array}{cc}
A_{c i} & B_{c i}
\end{array}\right]+} \\
\sum_{i=1}^{r-1} \sum_{q=i+1}^{r} h_{k(i)} h_{k(q)}\left[\begin{array}{cc}
A_{c i q} & B_{c i q}
\end{array}\right], \\
{\left[\begin{array}{ll}
C_{c}\left(h_{k}\right) & \left.D_{c}\left(h_{k}\right)\right]
\end{array}\right]=\sum_{i=1}^{r} h_{k(i)}\left[\begin{array}{ll}
C_{c i} & D_{c i}
\end{array}\right]}
\end{gathered}
$$

sendo $A_{c i} \in \Re^{n \times n}, A_{c i q} \in \Re^{n \times n}, B_{c i} \in \Re^{n \times p}, B_{c i q} \in$ $\Re^{n \times p}, C_{c i} \in \Re^{m \times n}$ e $D_{c i} \in \Re^{m \times p}$. Como as matrizes da equação de saída, $C_{c}\left(h_{k}\right)$ e $D_{c}\left(h_{k}\right)$, dependem do parâmetro variante, nomeia-se este controlador de CDRS-TDP (Totalmente Dependente de Parâmetros).

Considerando a interconexão $u_{c, k}=y_{k}$ e $y_{c, k}=u_{k}$, temse o sistema em malha fechada:

$$
\left\{\begin{aligned}
x_{k+1}= & A\left(h_{k}\right) x_{k}+B\left(h_{k}\right) C_{c}\left(h_{k}\right) x_{c, k}+ \\
& B\left(h_{k}\right) D_{c}\left(h_{k}\right) C x_{k} \\
x_{c, k+1}= & A_{c}\left(h_{k}\right) x_{c, k}+B_{c}\left(h_{k}\right) C x_{k}
\end{aligned}\right.
$$

Como anteriormente, tem-se

$$
\varsigma_{k+1}=\left(\mathbb{A}\left(h_{k}\right)+\mathbb{B}\left(h_{k}\right) \mathbb{K}\left(h_{k}\right)\right)
$$

com

$$
\begin{aligned}
& \mathbb{A}\left(h_{k}\right)=\left[\begin{array}{cc}
A\left(h_{k}\right) & 0 \\
B_{c}\left(h_{k}\right) C & A_{c}\left(h_{k}\right)
\end{array}\right], \quad \mathbb{B}\left(h_{k}\right)=\left[\begin{array}{c}
B\left(h_{k}\right) \\
0
\end{array}\right] \\
& \text { e } \mathbb{K}\left(h_{k}\right)=\left[\begin{array}{ll}
D_{c}\left(h_{k}\right) C & C_{c}\left(h_{k}\right)
\end{array}\right] \text {. }
\end{aligned}
$$

Observa-se de (9) que a matriz $\mathbb{K}\left(h_{k}\right)$ pode ser vista como uma realimentação dependente de parâmetros dos estados do sistema aumentado. 
Observação 1 A estrutura diferenciada (7), proposta para $A_{c}\left(h_{k}\right)$ e $B_{c}\left(h_{k}\right)$, é necessária para a obtenção dos parâmetros do CDRS-TDP a partir das condições de estabilização que serão propostas sob a forma de LMIs na seção 4. Conforme será explicado posteriormente, a utilização de uma estrutura simplificada, utilizando (4) e (8) conjuntamente, inviabiliza este procedimento de obtenção dos parâmetros do compensador.

Observação 2 Pode-se verificar a partir de (4), (7) e (8) que o CDRS-TDP apresenta maior complexidade de implementação do que o CDRS-PDP. A complexidade de implementação dos compensadores propostos está relacionada ao número de operações que devem ser realizadas à cada iteração e ao número de parâmetros que devem estar disponíveis on-line para obter o valor do controle, $u_{k}$. A verificação desta complexidade é de interesse, por exemplo, em aplicações críticas embarcadas onde restrições econômicas e de consumo de energia requerem a utilização de dispositivos para cálculo com limitações em termos de processamento e de disponibilidade de memória (Arzen and Cervin, 2005).

\section{RESULTADOS PRELIMINARES}

Para o estudo de estabilidade e a garantia de um certo desempenho temporal para o sistema em malha fechada, considera-se uma Função de Lyapunov Dependente de Parâmetro (FLDP) tal que $V\left(\varsigma_{k}, h_{k}\right): \Re^{2 n} \times \Xi \rightarrow \Re^{+}$, visando reduzir o conservadorismo inerente à utilização de Funções de Lyapunov constantes ou independentes de parâmetros.

Definição 1: Considere um escalar não negativo $\lambda \in(0,1]$. A origem do sistema descrito em (5) ou (9) é robustamente assintoticamente estável, com coeficiente de contratividade $\lambda$ (Milani and Coelho, 2002), se

$$
\begin{gathered}
\Delta V_{\lambda}\left(\varsigma_{k}, h_{k}\right) \triangleq V\left(\varsigma_{k+1}, h_{k+1}\right)-\lambda V\left(\varsigma_{k}, h_{k}\right)<0 \\
\forall \varsigma_{k} \in \Re^{n}, \quad \varsigma_{k} \neq 0 \quad \text { e } \forall h_{k} \in \Xi .
\end{gathered}
$$

Na sequência, assume-se a FLDP da seguinte forma

$$
V\left(\varsigma_{k}, h_{k}\right)=\varsigma_{k}^{\prime} \mathbb{Q}^{-1}\left(h_{k}\right) \varsigma_{k}
$$

$\operatorname{com} \mathbb{Q}\left(h_{k}\right)=\sum_{i=1}^{r} h_{k(i)} \mathbb{Q}_{i}, \quad \mathbb{Q}_{i}=\mathbb{Q}_{i}^{\prime}>0, \quad h_{k} \in \Xi$.

Então, considerando que (5) e (9) podem ser escritos sob a forma

$$
\varsigma_{k+1}=\mathbb{A}_{M F}\left(h_{k}\right) \varsigma_{k}
$$

com $\mathbb{A}_{M F}\left(h_{k}\right)=\mathbb{A}\left(h_{k}\right)+\mathbb{B}\left(h_{k}\right) \mathbb{K}$ para o CDRS-PDP e $\mathbb{A}_{M F}\left(h_{k}\right)=\mathbb{A}\left(h_{k}\right)+\mathbb{B}\left(h_{k}\right) \mathbb{K}\left(h_{k}\right)$ para o CDRS-TDP. Desta forma obtém-se

$$
\Delta V_{\lambda}\left(\varsigma_{k}, h_{k}\right)=\varsigma_{k}^{\prime} \mathcal{M}\left(h_{k+1}, h_{k}\right) \varsigma_{k}<0
$$

sendo

$$
\begin{gathered}
\mathcal{M}\left(h_{k+1}, h_{k}\right)=\mathbb{A}_{M F}^{\prime}\left(h_{k}\right) \mathbb{Q}^{-1}\left(h_{k+1}\right) \mathbb{A}_{M F}\left(h_{k}\right)- \\
\lambda \mathbb{Q}^{-1}\left(h_{k}\right) .
\end{gathered}
$$

Note que (13) é verificada se e somente se $\mathcal{M}\left(h_{k+1}, h_{k}\right)<0$, ou, de forma equivalente (por complemento de Schur) (Boyd et al., 1994)

$$
\left[\begin{array}{cc}
-\mathbb{Q}\left(h_{k+1}\right) & \mathbb{A}_{M F}\left(h_{k}\right) \\
* & -\lambda \mathbb{Q}^{-1}\left(h_{k}\right)
\end{array}\right]<0
$$

Para a obtenção de condições que permitam a síntese dos controladores dinâmicos com as estruturas desejadas, pré e pós multiplica-se (14) por $\operatorname{diag}\left(\left[\begin{array}{ll}I & \mathbb{U}\end{array}\right]\right)$ e sua transposta, considerando o fato que, para qualquer matriz invertível $\mathbb{U} \in \Re^{2 n \times 2 n}$, tem-se (Daafouz and Bernussou, 2001)

$$
\begin{gathered}
\left(\mathbb{U}-\mathbb{Q}\left(h_{k}\right)\right)^{\prime} \mathbb{Q}\left(h_{k}\right)^{-1}\left(\mathbb{U}-\mathbb{Q}\left(h_{k}\right)\right) \geq 0 \Leftrightarrow \\
-\mathbb{U}^{\prime} \mathbb{Q}\left(h_{k}\right)^{-1} \mathbb{U} \leq \mathbb{Q}\left(h_{k}\right)-\mathbb{U}-\mathbb{U}^{\prime} .
\end{gathered}
$$

Portanto, uma condição suficiente para a verificação de (14) pode ser formulada como a desigualdade matricial dependente de parâmetros:

$$
\left[\begin{array}{cc}
-\mathbb{Q}\left(h_{k+1}\right) & \mathbb{A}_{M F}\left(h_{k}\right) \mathbb{U} \\
* & \lambda\left(\mathbb{Q}\left(h_{k}\right)-\mathbb{U}-\mathbb{U}^{\prime}\right)
\end{array}\right]<0
$$

A partir de (15) podemos então estabelecer as condições para análise de estabilidade e do desempenho temporal associado ao coeficiente de contratividade $\lambda$, descritas em função dos vértices dos politopos de matrizes, associadas às duas estruturas de controladores dinâmicos sob investigação.

\section{Lema 1 Estabilidade CASO 1 - CDRS-PDP}

Sejam $A_{c i}, B_{c i}, C_{c}$ e $D_{c}$ matrizes conhecidas que formam o controlador (3). Para um dado escalar real $\lambda \in(0,1]$, considere a existência de matrizes simétricas definidas positivas $\mathbb{Q}_{i} \in \Re^{2 n \times 2 n}$ e da matriz $\mathbb{U} \in \Re^{2 n \times 2 n}$, satisfazendo:

$$
\mathbb{M}_{1 j i}^{+}=\left[\begin{array}{cc}
-\mathbb{Q}_{j} & \mathbb{A}_{i} \mathbb{U}+\mathbb{B}_{i} \mathbb{K} \mathbb{U} \\
* & \lambda\left(\mathbb{Q}_{i}-\mathbb{U}-\mathbb{U}^{\prime}\right)
\end{array}\right]<0
$$

Então, a origem do sistema em malha fechada (5) é robustamente assintoticamente estável, com coeficiente de contratividade $\lambda$, para qualquer condição inicial $\varsigma_{0} \in \Re^{n}$. 
Prova: Para a demonstração considere:

$$
V\left(\varsigma_{k+1}, h_{k+1}\right)=\varsigma_{k+1}^{\prime} \mathbb{Q}^{-1}\left(h_{k+1}\right) \varsigma_{k+1}
$$

A partir de (16), para cada $i$, multiplica-se a desigualdade correspondente $j=1, \ldots, r$ por $h_{k+1(j)}$ e soma-se. $\mathrm{Na}$ sequência multiplicam-se as desigualdades resultantes $i=1, \ldots, r$ por $h_{k(i)}$ e somam-se, obtendo-se:

$$
\sum_{i=1}^{r} h_{k(i)}\left(\sum_{j=1}^{r} h_{k+1(j)} \mathbb{M}_{1 j i}^{+}\right)<0 .
$$

Com base nas estruturas definidas em (2) e (4), e dos valores admissíveis aos parâmetros $\left(\sum_{i=1}^{r} h_{k(i)}=\right.$ $\left.1, h_{k(i)} \geq 0\right)$, pode-se reescrever:

$$
\left[\begin{array}{cc}
-\mathbb{Q}\left(h_{k+1}\right) & \left(\mathbb{A}\left(h_{k}\right)+\mathbb{B}\left(h_{k}\right) \mathbb{K}\right) \mathbb{U} \\
* & \lambda\left(\mathbb{Q}\left(h_{k}\right)-\mathbb{U}-\mathbb{U}^{\prime}\right)
\end{array}\right]<0 .
$$

que corresponde à condição de estabilidade apresentada em (15).

\section{Lema 2 Estabilidade CASO 2 - CDRS-TDP}

Sejam $A_{c i}, A_{c i q}, B_{c i}, B_{c i q}, C_{c i}$ e $D_{c i}$ matrizes conhecidas que formam o controlador (6). Para um dado escalar real $\lambda \in(0,1]$, considere a existência de matrizes simétricas definidas positivas $\mathbb{Q}_{i} \in \Re^{2 n \times 2 n}$ e da matriz $\mathbb{U} \in \Re^{2 n \times 2 n}$, satisfazendo:

$$
\begin{gathered}
\mathbb{M}_{2 j i}^{+}=\left[\begin{array}{cc}
-\mathbb{Q}_{j} & \left(\mathbb{A}_{i}+\mathbb{B}_{i} \mathbb{K}_{i}\right) \mathbb{U} \\
* & \lambda\left(\mathbb{Q}_{i}-\mathbb{U}-\mathbb{U}^{\prime}\right)
\end{array}\right]<0 \\
\forall i, j=1, \ldots, r \\
\mathbb{M}_{2 j i q}^{+}=\left[\begin{array}{cc}
-2 \mathbb{Q}_{j} & \left(\mathbb{A}_{i q}+\mathbb{B}_{i} \mathbb{K}_{q}+\mathbb{B}_{q} \mathbb{K}_{i}\right) \mathbb{U} \\
* & \lambda\left(\mathbb{Q}_{i}+\mathbb{Q}_{q}-2 \mathbb{U}-2 \mathbb{U}^{\prime}\right)
\end{array}\right]<0 \\
\forall j=1, \ldots, r, \quad \forall i=1, \ldots, r-1 \text { e } \forall q=i+1, \ldots, r
\end{gathered}
$$

Então, a origem do sistema em malha fechada (9) é robustamente assintoticamente estável, com coeficiente de contratividade $\lambda$, para qualquer condição inicial $\varsigma_{0} \in \Re^{n}$.

Prova: Para o caso totalmente dependente de parâmetros, considera-se a condição (15) com

$$
\mathbb{A}_{M F}\left(h_{k}\right)=\mathbb{A}\left(h_{k}\right)+\mathbb{B}\left(h_{k}\right) \mathbb{K}\left(h_{k}\right)
$$

Das estruturas matriciais definidas em (2) e (8) pode-se reescrever a parcela $\mathbb{A}_{M F}\left(h_{k}\right)$ como

$$
\mathbb{A}_{M F}\left(h_{k}\right)=\sum_{i=1}^{r} h_{k(i)}^{2} \underbrace{\left[\begin{array}{cc}
A_{i}+B_{i} D_{c i} C & B_{i} C_{c i} \\
B_{c i} C & A_{c i}
\end{array}\right]}_{\mathbb{A}_{i}+\mathbb{B}_{i} \mathbb{K}_{i}}+
$$

$$
\sum_{i=1}^{r-1} \sum_{q=i+1}^{r} h_{k(i)} h_{k(q)} \underbrace{\left[\begin{array}{cc}
\mathbf{M}_{\mathbf{a} \mathbf{i q}} & \mathbf{M}_{\mathbf{a} \mathbf{i q}} \\
B_{c i q} C & A_{c i q}
\end{array}\right]}_{\mathbb{A}_{i q}+\mathbb{B}_{i} \mathbb{K}_{q}+\mathbb{B}_{q} \mathbb{K}_{i}}
$$

onde $\mathbf{M}_{\mathbf{a} 1 \mathbf{i q}}=\left(A_{i}+A_{q}\right)+\left(B_{i} D_{c q}+B_{q} D_{c i}\right) C, \mathbf{M}_{\mathbf{a} 2 \mathbf{i q}}=$ $B_{i} C_{c q}+B_{q} C_{c i}$ e $\mathbb{A}_{i q}=\left[\begin{array}{cc}A_{i}+A_{q} & 0 \\ B_{c i q} C & A_{c i q}\end{array}\right]$.

De maneira análoga para os demais termos, pode-se reescrever (15) como

$$
\begin{gathered}
\sum_{j=1}^{r} \sum_{i=1}^{r} h_{k+1(j)} h_{k(i)}^{2} M_{2 j i}^{+}+ \\
\sum_{j=1}^{r} \sum_{i=1}^{r-1} \sum_{q=i+1}^{r} h_{k+1(j)} h_{k(i)} h_{k(q)} M_{2 j i q}^{+}<0
\end{gathered}
$$

Então, considerando-se os valores admissíveis dos parâmetros $\left(\sum_{i=1}^{r} h_{k(i)}=1, h_{k(i)} \geq 0\right)$, as condições estabelecidas pelo lema garantem que (19) é verificada.

Observa-se de (16), (17) e (18), que a matriz $\mathbb{U}$ é obrigatoriamente invertível. Além disso, à FLDP (11) utilizada para a verificação da estabilidade e desempenho do sistema em malha fechada, pode-se associar conjuntos de tipo Lyapunov, dados por (Hu et al., 2002; Castelan et al., 2010):

$$
\begin{gathered}
\mathcal{L}_{V}(\gamma)=\left\{\varsigma_{k} \in \Re^{2 n} ; \varsigma_{k}^{\prime} \mathbb{Q}^{-1}\left(h_{k}\right) \varsigma_{k} \leq \gamma, \gamma \in \Re^{+}\right\} \\
\triangleq \bigcap_{h_{k} \in \Xi}\left\{\mathscr{E}\left(\mathbb{Q}^{-1}\left(h_{k}\right), \gamma\right)\right\}=\bigcap_{i \in\{1, \ldots r\}} \mathscr{E}\left(\mathbb{Q}_{i}^{-1}, \gamma\right)
\end{gathered}
$$

em que $\mathscr{E}\left(\mathbb{Q}_{i}^{-1}, \gamma\right)=\left\{\varsigma_{k} \in \Re^{2 n} ; \varsigma_{k}^{\prime} \mathbb{Q}_{i}^{-1} \varsigma_{k} \leq \gamma\right\}$ são conjuntos elipsoidais.

Como consequência do Lema 1 e do Lema $2, \mathcal{L}_{V}(\gamma)=$ $\bigcap_{i} \mathscr{E}\left(\mathbb{Q}_{i}^{-1}, \gamma\right)$ é um conjunto robustamente $\lambda$-contrativo (com respeito as trajetórias do sistema (5) ou (9)); para maiores detalhes, veja por exemplo, (Corso et al., 2009). Esta noção de conjunto contrativo, que garante que toda trajetória que inicia em $\mathcal{L}_{V}(\gamma)$ permanece neste domínio e converge assintoticamente para a origem, será utilizada mais adiante para caracterizar domínios de estabilidade quando o modelo LPV representa um sistema não linear num domínio de validade local.

\section{RESULTADOS PRINCIPAIS}

Para obtenção das condições de síntese dos controladores sob investigação, definem-se, da mesma forma que em (Castelan et al., 2010), as seguintes matrizes adaptadas de (Scherer et al., 1997): 
$\mathbb{U}=\left[\begin{array}{cc}X & N \\ Z & \bullet\end{array}\right], \mathbb{U}^{-1}=\left[\begin{array}{cc}Y & M \\ W & \bullet\end{array}\right]$ e $\Theta=\left[\begin{array}{cc}Y & I \\ W & 0\end{array}\right]$

Para estas matrizes, tem-se

$$
\mathbb{U} \Theta=\left[\begin{array}{cc}
I & X \\
0 & Z
\end{array}\right] \Rightarrow \Theta^{\prime} \mathbb{U} \Theta=\left[\begin{array}{cc}
Y^{\prime} & T^{\prime} \\
I & X
\end{array}\right]
$$

em que, por construção:

$$
T^{\prime}=Y^{\prime} X+W^{\prime} Z
$$

Ainda, de $\mathbb{U} \mathbb{U}^{-1}=I$ e $\mathbb{U}^{-1} \mathbb{U}=I$, tem-se

$$
X Y+N W=Y X+M Z=I .
$$

Além disso, particionando $\mathbb{Q}_{i}=\left[\begin{array}{cc}Q_{11 i} & Q_{12 i} \\ * & Q_{22 i}\end{array}\right]$, podese definir:

$$
\Theta^{\prime} \mathbb{Q}_{i} \Theta=\left[\begin{array}{cc}
\bar{Q}_{11 i} & \bar{Q}_{12 i} \\
* & \bar{Q}_{22 i}
\end{array}\right]
$$

Os teoremas descritos a seguir estabelecem condições de estabilização robusta, com desempenho temporal associado ao coeficiente de contratividade $\lambda$, sob a forma de LMIs. Estas condições são obtidas a partir das condições (16), (17) e (18).

\subsection{CDRS Parcialmente Dependente de Parâmetros}

Teorema 1 Dado $\lambda \in(0,1]$, considere que existem matrizes simétricas definidas positiva $\bar{Q}_{11 i}, \bar{Q}_{22 i}$, e matrizes $\bar{Q}_{12 i}, \hat{A}_{i}, X, Y, T, \hat{B}_{i}, \hat{C}$ e $\hat{D}$, satisfazendo as seguintes condições:

$$
\left[\begin{array}{cccc}
-\bar{Q}_{11 j} & -\bar{Q}_{12 j} & Y^{\prime} A_{i}+\hat{B}_{i} C & \hat{A}_{i} \\
* & -\bar{Q}_{22 j} & A_{i}+B_{i} \hat{D} C & \mathbf{M}_{\mathbf{b} \mathbf{i}} \\
* & * & \mathbf{M}_{\mathbf{b 4 i}} & \mathbf{M}_{\mathbf{b 2 i}} \\
* & * & * & \mathbf{M}_{\mathbf{b 3 i}}
\end{array}\right]<0
$$

nas quais:

$$
\begin{aligned}
& \mathbf{M}_{\mathbf{b} 1 \mathbf{i}}=A_{i} X+B_{i} \hat{C}, \\
& \mathbf{M}_{\mathbf{b} \mathbf{i}}=\lambda\left(\bar{Q}_{12 i}-T^{\prime}-I\right), \\
& \mathbf{M}_{\mathbf{b} 3 \mathbf{i}}=\lambda\left(\bar{Q}_{22 i}-X^{\prime}-X\right), \\
& \mathbf{M}_{\mathbf{b} 4 \mathbf{i}}=\lambda\left(\bar{Q}_{11 i}-Y^{\prime}-Y\right) .
\end{aligned}
$$

Seja $W \in \Re^{n \times n}$ uma matriz não singular qualquer, determine:

$$
Z=\left(W^{\prime}\right)^{-1}\left(T^{\prime}-Y^{\prime} X\right)
$$

Então, as matrizes do controlador CDRS-PDP (3) obtidas por

$$
\begin{aligned}
D_{c}= & \hat{D} \\
C_{c}= & \left(\hat{C}-D_{c} C X\right) Z^{-1} \\
B_{c i}= & \left(W^{\prime}\right)^{-1}\left(\hat{B}_{i}-Y^{\prime} B_{i} D_{c}\right) \\
A_{c i}= & \left(W^{\prime}\right)^{-1}\left(\hat{A}_{i}-Y^{\prime} A_{i} X-Y^{\prime} B_{i} D_{c} C X-\right. \\
& \left.Y^{\prime} B_{i} C_{c} Z-W^{\prime} B_{c i} C X\right) Z^{-1}
\end{aligned}
$$

são tais que a origem do sistema em malha fechada (5) é robustamente assintoticamente estável com coeficiente de contratividade $\lambda$.

Prova: Multiplica-se a condição de estabilidade (16) do Lema 1, à esquerda por $\operatorname{diag}\left[\Theta^{\prime} \Theta^{\prime}\right]$ e à direita pela sua transposta, conforme abaixo exposto:

$$
\left[\begin{array}{cc}
\Theta^{\prime} & 0 \\
0 & \Theta^{\prime}
\end{array}\right] M_{1 j i}^{+}\left[\begin{array}{cc}
\Theta & 0 \\
0 & \Theta
\end{array}\right]<0 .
$$

Desenvolvendo o produto e efetuando a substituição de variáveis

$$
\begin{aligned}
\hat{A}_{i}= & W^{\prime} A_{c i} Z+W^{\prime} B_{c i} C X+Y^{\prime} B_{i} C_{c} Z+ \\
& Y^{\prime}\left(A_{i}+B_{i} D_{c} C\right) X \\
\hat{B}_{i}= & Y^{\prime} B_{i} D_{c}+W^{\prime} B_{c i} \\
\hat{C}= & D_{c} C X+C_{c} Z \\
\hat{D}= & D_{c}
\end{aligned}
$$

verifica-se a equivalência das desigualdades (20) e (16).

Com vistas à sintese do controlador CDRS-PDP, a LMI (20) proposta no teorema anterior pode ser utilizada para formular problemas de programação convexa, podendo-se adicionar outras restrições e/ou reformular o problema para tratar outros critérios, como custo garantido e controle $H_{\infty}$.

\subsection{CDRS Totalmente Dependente de Pa- râmetros}

Teorema 2 Dado $\lambda \in(0,1]$, considere que existem matrizes simétricas definidas positiva $\bar{Q}_{11 i}, \bar{Q}_{22 i}$, e matrizes $\bar{Q}_{12 i}, \hat{A}_{i}, \hat{A}_{i q}, X, Y, T, \hat{B}_{i}, \hat{B}_{i q}, \hat{C}_{i}$ e $\hat{D}_{i}$, satisfazendo as seguintes condições:

$$
\left[\begin{array}{cccc}
-\bar{Q}_{11 j} & -\bar{Q}_{12 j} & Y^{\prime} A_{i}+\hat{B}_{i} C & \hat{A}_{i} \\
* & -\bar{Q}_{22 j} & A_{i}+B_{i} \hat{D}_{i} C & \mathbf{M}_{\mathbf{c 1 i}} \\
* & * & \lambda\left(\bar{Q}_{11 i}-Y^{\prime}-Y\right) & \mathbf{M}_{\mathbf{c 2 i}} \\
* & * & * & \mathbf{M}_{\mathbf{c 3 i}}
\end{array}\right]<0
$$




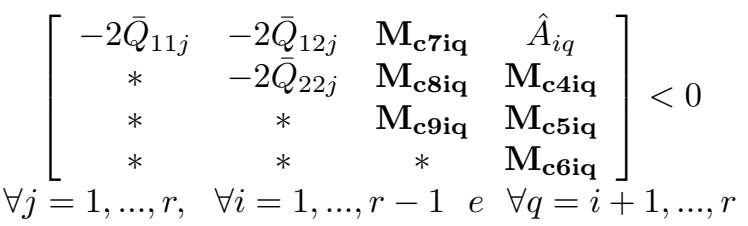

nas quais:

$$
\begin{aligned}
& \mathbf{M}_{\mathbf{c 1 i}}=A_{i} X+B_{i} \hat{C}_{i}, \\
& \mathbf{M}_{\mathbf{c} \mathbf{i} \mathbf{i}}=\lambda\left(\bar{Q}_{12 i}-T^{\prime}-I\right), \\
& \mathbf{M}_{\mathbf{c} \mathbf{3} \mathbf{i}}=\lambda\left(\bar{Q}_{22 i}-X^{\prime}-X,\right) \\
& \mathbf{M}_{\mathbf{c} \mathbf{i}}=\left(A_{i}+A_{q}\right) X+B_{i} \hat{C}_{q}+B_{q} \hat{C}_{i}, \\
& \mathbf{M}_{\mathbf{c 5 i}}=\lambda\left(\bar{Q}_{12 i}+\bar{Q}_{12 q}-2 T^{\prime}-2 I\right), \\
& \mathbf{M}_{\mathbf{c 6 i}}=\lambda\left(\bar{Q}_{22 i}+\bar{Q}_{22 q}-2 X^{\prime}-2 X\right), \\
& \mathbf{M}_{\mathbf{c} \mathbf{i} \mathbf{i}}=Y^{\prime}\left(A_{i}+A_{q}\right)+\hat{B}_{i q} C, \\
& \mathbf{M}_{\mathbf{c} \mathbf{i} \mathbf{i}}=A_{i}+A_{q}+\left(B_{i} \hat{D}_{q}+B_{q} \hat{D}_{i}\right) C, \\
& \mathbf{M}_{\mathbf{c} \mathbf{9} \mathbf{i}}=\lambda\left(\bar{Q}_{11 i}+\bar{Q}_{11 q}-2 Y^{\prime}-2 Y\right) .
\end{aligned}
$$

Seja $W \in \Re^{n \times n}$ uma matriz não singular qualquer, determine $Z$ a partir de (21). Então, as matrizes do controlador CDRS-TDP (6) obtidas por

$$
\begin{aligned}
D_{c i}= & \hat{D}_{i} \\
B_{c i}= & \left(W^{\prime}\right)^{-1}\left(\hat{B}_{i}-Y^{\prime} B_{i} D_{c i}\right) \\
C_{c i}= & \left(\hat{C}_{i}-D_{c i} C X\right) Z^{-1} \\
A_{c i}= & \left(W^{\prime}\right)^{-1}\left(\hat{A}_{i}-Y^{\prime} A_{i} X-Y^{\prime} B_{i} D_{c i} C X-\right. \\
& \left.Y^{\prime} B_{i} C_{c i} Z-W^{\prime} B_{c i} C X\right) Z^{-1} \\
B_{c i q}= & \left(W^{\prime}\right)^{-1}\left[\hat{B}_{i q}-Y^{\prime}\left(B_{i} D_{c q}+B_{q} D_{c i}\right)\right] \\
A_{c i q}= & \left(W^{\prime}\right)^{-1}\left[\hat{A}_{i q}-Y^{\prime}\left(A_{i}+A_{q}\right) X-\right. \\
& Y^{\prime}\left(B_{i} D_{c q}+B_{q} D_{c i}\right) C X-Y^{\prime}\left(B_{i} C_{c q}+\right. \\
& \left.\left.B_{q} C_{c i}\right) Z-W^{\prime} B_{c i q} C X\right] Z^{-1}
\end{aligned}
$$

são tais que a origem do sistema em malha fechada é robustamente assintoticamente estável com coeficiente de contratividade $\lambda$.

Prova: Multiplica-se as condições de estabilidade (17) e (18) do Lema 2, à esquerda por $\operatorname{diag}\left[\Theta^{\prime} \Theta^{\prime}\right]$ e à direita pela sua transposta, conforme abaixo exposto

$$
\begin{gathered}
{\left[\begin{array}{cc}
\Theta^{\prime} & 0 \\
0 & \Theta^{\prime}
\end{array}\right] M_{2 j i}^{+}\left[\begin{array}{cc}
\Theta & 0 \\
0 & \Theta
\end{array}\right]<0,} \\
{\left[\begin{array}{cc}
\Theta^{\prime} & 0 \\
0 & \Theta^{\prime}
\end{array}\right] M_{2 j i q}^{+}\left[\begin{array}{cc}
\Theta & 0 \\
0 & \Theta
\end{array}\right]<0 .}
\end{gathered}
$$

Desenvolvendo os produtos e efetuando a substituição de variáveis

$$
\begin{aligned}
\hat{A}_{i}= & W^{\prime} A_{c i} Z+W^{\prime} B_{c i} C X+Y^{\prime} B_{i} C_{c i} Z+ \\
& Y^{\prime}\left(A_{i}+B_{i} D_{c i} C\right) X \\
\hat{A}_{i q}= & Y^{\prime}\left(A_{i}+A_{q}\right) X+Y^{\prime}\left(B_{i} D_{c q}+\right. \\
& \left.B_{q} D_{c i}\right) C X+W^{\prime} B_{c i q} C X+Y^{\prime}\left(B_{i} C_{c q}+\right. \\
& \left.B_{q} C_{c i}\right) Z+W^{\prime} A_{c i q} Z \\
\hat{B}_{i}= & Y^{\prime} B_{i} D_{c i}+W^{\prime} B_{c i} \\
\hat{B}_{i q}= & Y^{\prime}\left(B_{i} D_{c q}+B_{q} D_{c i}\right)+W^{\prime} B_{c i q} \\
\hat{C}_{i}= & D_{c i} C X+C_{c i} Z \\
\hat{D}_{i}= & D_{c i}
\end{aligned}
$$

verifica-se a equivalência das desigualdades (22) e (23) com (17) e (18).

A proposição deste teorema permite, tal como o Teorema 1, a formulação de problemas de programação convexa. É importante destacar também que o CDRS-PDP pode ser visto como um caso particular do CDRS-TDP, eliminando-se a condição (23) do Teorema 2, fazendo $\hat{C}_{i}=\hat{C}$ e $\hat{D}_{i}=\hat{D}$ para a condição (22), e utilizando a mesma estrutura matricial representada em (4).

Observação 3 Uma estrutura simplificada de CDRSTDP consiste em considerar as matrizes de estado e de entrada do compensador com a estrutura (4) e as matrizes associadas à saída do compensador com a estrutura (8). Neste caso, tem-se, em particular:

$$
\begin{gathered}
\mathbb{A}_{M F}\left(h_{k}\right)=\sum_{i=1}^{r} h_{k(i)}\left[\begin{array}{cc}
A_{i} & 0 \\
B_{c i} C & A_{c i}
\end{array}\right]+ \\
\sum_{i=i}^{r} \sum_{q=1}^{r} h_{k(i)} h_{k(q)}\left[\begin{array}{c}
B_{i} \\
0
\end{array}\right]\left[\begin{array}{ll}
D_{c q} C & C_{c q}
\end{array}\right]
\end{gathered}
$$

Então, redefinindo $\mathbb{A}_{i q}=\left[\begin{array}{cc}A_{i}+A_{q} & 0 \\ \left(B_{c i}+B_{c q}\right) C & A_{c i}+A_{c q}\end{array}\right]$ na demonstração do Lema 2 , é possível verificar que as LMIs (17) e (18) também permitem concluir sobre a estabilidade do compensador com estrutura simplificada.

Note, entretanto, que o desenvolvimento para esta estrutura simplificada dos produtos utilizados na demonstração do Teorema 2 , leva à substituição das variáveis $\hat{A}_{i q} \mathrm{e}$ $\hat{B}_{i q}$ mostrada a seguir, a qual impossibilita a construção 
das matrizes $A_{c i}$ e $B_{c i}$ :

$$
\begin{aligned}
\hat{A}_{i q}= & Y^{\prime}\left(A_{i}+A_{q}\right) X+Y^{\prime}\left(B_{i} D_{c q}+B_{q} D_{c i}\right) C X+ \\
& W^{\prime}\left(B_{c i}+B_{c q}\right) C X+Y^{\prime}\left(B_{i} C_{c q}+\right. \\
& \left.B_{q} C_{c i}\right) Z+W^{\prime}\left(A_{c i}+A_{c q}\right) Z \\
\hat{B}_{i q}= & Y^{\prime}\left(B_{i} D_{c q}+B_{q} D_{c i}\right)+W^{\prime}\left(B_{c i}+B_{c q}\right)
\end{aligned}
$$

\subsection{Estabilização Local num Domínio de Validade do Sistema}

Os resultados apresentados anteriormente consideram que o sistema com parâmetros variantes (1) é válido para todo $\Re^{n}$, e, desta forma, que a estabilidade e o desempenho serão válidos em todo o espaço de estados do sistema em malha fechada (12).

Entretanto, em vários problemas práticos o modelo (1) é válido apenas localmente (regionalmente), e descreve o comportamento do sistema somente num subconjunto do espaço de estados, $\mathcal{V}_{0} \subset \Re^{n}$, denominado de domínio de validade do modelo. Nestes casos, para garantir a estabilidade e o desempenho do sistema controlado via uma das estruturas de CDRS apresentadas, pode-se incluir uma condição adicional às condições de estabilidade dos Lemas 1 e 2, com o objetivo de garantir a inclusão de um domínio $\lambda$-contrativo $\mathcal{L}_{V}=\mathcal{L}_{V}(1)=\bigcap_{i} \mathscr{E}\left(\mathbb{Q}_{i}^{-1}, 1\right)$ na região de validade do sistema em malha fechada, i.e., $\mathcal{L}_{V} \subseteq \mathcal{X}_{0}$ com

$$
\mathcal{X}_{0} \triangleq\left\{\varsigma_{k}=\left[\begin{array}{c}
x_{k} \\
x_{c, k}
\end{array}\right] \in \Re^{2 n} ; \quad x \in \mathcal{V}_{0}, \quad \forall x_{c} \in \Re^{n}\right\} .
$$

Desta forma, garante-se que para qualquer condição inicial $\varsigma_{0} \in \mathcal{L}_{V}$, a trajetória correspondente evolui no interior de $\mathcal{L}_{V}$ e tende assintoticamente à origem. Portanto, a evolução das trajetórias dos estados da planta é confinada no interior do domínio de validade $\mathcal{V}_{0}$.

Seja, por exemplo, o domínio de validade definido por um conjunto poliedral

$$
\begin{aligned}
\mathcal{V}_{0} & =C o\left\{v_{\tau} \in \Re^{n}, \quad \tau=1, \ldots, n_{\tau}\right\} \\
& =\left\{x_{k} \in \Re^{n} ; \quad\left|L x_{k}\right| \leq \varrho\right\},
\end{aligned}
$$

em que $L \in \Re^{n \times n}$ e $\varrho \in \Re^{n}$, com $\varrho_{i} \geq 0$. A extensão de $\mathcal{V}_{0}$ ao espaço de estados do sistema aumentado, denotada $\mathcal{X}_{0}$, é dada por

$$
\begin{aligned}
\mathcal{X}_{0} & =C o\left\{v_{\tau}^{a} \in \Re^{2 n} ; v_{\tau}^{a}=\left[\begin{array}{c}
v_{\tau} \\
0
\end{array}\right], \tau=1, \ldots, n_{\tau}\right\} \\
& =\left\{\varsigma_{k} \in \Re^{2 n} ; \quad\left|L_{a} \varsigma_{k}\right| \leq \varrho\right\}
\end{aligned}
$$

com $L_{a}=\left[\begin{array}{ll}L & 0\end{array}\right]$. A inclusão $\mathcal{L}_{V} \subseteq \mathcal{X}_{0}$ pode ser descrita pela seguinte restrição convexa (Boyd et al., 1994; Castelan et al., 2010)

$$
\begin{gathered}
{\left[\begin{array}{cc}
-\mathbb{Q}_{i}+\mathbb{U}^{\prime}+\mathbb{U} & \mathbb{U}^{\prime} L_{a(l)}^{\prime} \\
* & \Lambda_{(l)}^{2}
\end{array}\right] \geq 0} \\
\forall i=1, \ldots, r \text { e } \\
\forall l=1, \ldots, 2 n
\end{gathered}
$$

equivalente a

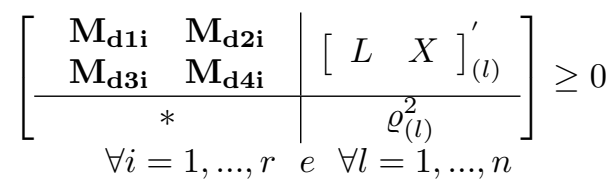

nas quais:

$$
\begin{aligned}
& \mathbf{M}_{\mathbf{d} 1 \mathbf{i}}=-\bar{Q}_{11 i}+Y^{\prime}+Y \\
& \mathbf{M}_{\mathbf{d} 2 \mathbf{i}}=-\bar{Q}_{12 i}+T^{\prime}+I \\
& \mathbf{M}_{\mathbf{d} 3 \mathbf{i}}=-\bar{Q}_{12 i}^{\prime}+T+I \\
& \mathbf{M}_{\mathbf{d} 4 \mathbf{i}}=-\bar{Q}_{22 i}+X^{\prime}+X
\end{aligned}
$$

Portanto, com base nas condições dos Lemas 1 e 2, e na condição estabelecida em (24), é possível apresentar os seguintes resultados de estabilidade e desempenho locais.

Corolário 1 Sejam $A_{c i}, B_{c i}, C_{c}$ e $D_{c}$ matrizes conhecidas que formam o controlador (3). Para um dado escalar real $\lambda \in(0,1]$, considere a existência de matrizes simétricas definidas positivas $\mathbb{Q}_{i} \in \Re^{2 n \times 2 n}$ e da matriz $\mathbb{U} \in \Re^{2 n \times 2 n}$, satisfazendo as condições definidas em (16) e (24). Então, o conjunto $\mathcal{L}_{V} \triangleq \bigcap_{i}\left\{\mathscr{E}\left(\mathbb{Q}_{i}^{-1}\right)\right\}=$ $\mathscr{E}\left(\mathbb{Q}^{-1}\left(h_{k}\right)\right)$ é robustamente absolutamente $\lambda$-contrativo e tal que $\mathcal{L}_{V} \subseteq \mathcal{X}_{0}$.

Corolário 2 Sejam $A_{c i}, A_{c i q}, B_{c i}, B_{c i q}, C_{c i}$ e $D_{c i}$ matrizes conhecidas que formam o controlador (6). Para um dado escalar real $\lambda \in(0,1]$, considere a existência de matrizes simétricas definidas positivas $\mathbb{Q}_{i} \in \Re^{2 n \times 2 n}$ e da matriz $\mathbb{U} \in \Re^{2 n \times 2 n}$, satisfazendo as condições definidas em (17), (18) e (24). Então, o conjunto $\mathcal{L}_{V} \triangleq$ $\bigcap_{i}\left\{\mathscr{E}\left(\mathbb{Q}_{i}^{-1}\right)\right\}=\mathscr{E}\left(\mathbb{Q}^{-1}\left(h_{k}\right)\right)$ é robustamente absolutamente $\lambda$-contrativo e tal que $\mathcal{L}_{V} \subseteq \mathcal{X}_{0}$.

Portanto, a partir dos Colorários 1 e 2, garante-se que a origem do sistema (5) ou (9) será assintoticamente estável para qualquer condição inicial $\varsigma_{0} \in \mathcal{L}_{V}$, e, seguramente, as trajetórias que emanam de $\varsigma_{0}$ permanecem em $\mathcal{X}_{0}$. Como consequencia, pode-se utilizar as condições dos Teoremas 1 e 2, conjuntamente com a condição de inclusão (25) para estabelecer os resultados de estabilização local. 
Para a síntese dos ganhos dos controladores, o domínio $\mathcal{L}_{V}$ deve ser o maior possível. Assim, para a otimização do domínio contrativo $\mathcal{L}_{V}$, é possível adotar, como em (Corso et al., 2009), um conjunto modelo para síntese. Tal conjunto pode ser adotado com a forma da própria região de validade. $\mathrm{O}$ objetivo consiste em maximizar o fator escalar $\beta$ tal que a inclusão $\beta \mathcal{X}_{0} \subseteq \mathcal{L}_{V}$ também seja verificada. Considerando $\mu=1 / \beta^{2}$, a condição pode ser descrita por

$$
\begin{array}{rl}
{\left[\begin{array}{cc}
\mu & v_{\sigma}^{\prime} \\
* & \mathbb{Q}_{i}
\end{array}\right]} & \geq 0 \\
\forall i=1, \ldots, r & e \quad \forall \sigma=1, \ldots, n_{\tau}
\end{array}
$$

equivalente a

$$
\begin{gathered}
{\left[\begin{array}{ccc}
\mu & v_{\tau}^{\prime} Y & v_{\tau}^{\prime} \\
* & \bar{Q}_{11 i} & \bar{Q}_{12 i} \\
* & * & \bar{Q}_{22 i}
\end{array}\right] \geq 0} \\
\forall i=1, \ldots, r \quad e \quad \forall \tau=1, \ldots, n_{\tau}
\end{gathered}
$$

Desta forma, para a síntese do CDRS-PDP aplicado a sistemas lineares com parâmetros variantes, considerando um domínio de validade $\mathcal{V}_{0}$, propõe-se o seguinte problema de programação convexa:

$$
\begin{gathered}
\min _{\bar{Q}_{11 i}, \bar{Q}_{12 i}, \bar{Q}_{222}, \hat{A}_{i},} \\
\quad X, Y, T, \hat{B}_{i}, \hat{C}, \hat{D} \\
\text { sujeito a }
\end{gathered}
$$$$
\text { LMIs (20), (25) e (26), }
$$

e, para o CDRS-TDP:

$$
\begin{gathered}
\min _{\bar{Q}_{11 i}, \bar{Q}_{12 i}, \bar{Q}_{22 i}, \hat{A}_{i}, \hat{A}_{i q},} \mu \\
X, Y, T, \hat{B}_{i}, \hat{B}_{i q}, \hat{C}_{i}, \hat{D}_{i} \\
\text { sujeito } \quad a
\end{gathered}
$$$$
\text { LMIs (22), (23), (25) e (26). }
$$

Nota-se que as condições adicionais não implicam em maior complexidade de implementação dos compensadores dinâmicos, apenas alteram a complexidade numérica de solução do algoritmo.

Observação 4 A complexidade numérica para a solução de problemas baseados em LMIs está relacionada ao número escalar de variáveis de decisão e ao número de linhas das LMIs consideradas (Leite et al., 2004). Assim, pode-se verificar que o algoritmo (28) é numericamente mais complexo que o (27). Entretanto, é importante destacar que a complexidade numérica está relacionada somente aos cálculos realizados off-line. Devido à grande capacidade de cálculo disponível atualmente, mesmo em computadores pessoais, a complexidade numérica não coloca restrição forte em relação à utilização do CDRS-TDP frente ao CDRS-PDP, mesmo para sistemas de maior ordem.

\section{APLICAÇÃO A UM SISTEMA FUZZY TAKAGI-SUGENO}

\subsection{Apresentação do Sistema e Modelo Fuzzy}

Sejam as equações de movimento descritas em (Cannon, 1967), representando o problema de equilibrar um pêndulo invertido em um carro

$$
\begin{aligned}
& \dot{x}_{1}=x_{2} \\
& \dot{x}_{2}=\frac{g \operatorname{sen}\left(x_{1}\right)-a m l x_{2}^{2} \operatorname{sen}\left(2 x_{1}\right) / 2-a \cos \left(x_{1}\right) u}{4 l / 3-a m l \cos ^{2}\left(x_{1}\right)}
\end{aligned}
$$

sendo: $x_{1}$ o ângulo (em radianos) do pêndulo em relação a vertical; $x_{2}$ a velocidade angular (em radianos por segundo); $g=9.8 \mathrm{~m} / \mathrm{s}^{2}$ a aceleração da gravidade; $m$ a massa (em quilogramas) do pêndulo; $M$ a massa (em quilogramas) do carro; $2 l$ o comprimento (em metros) do pêndulo e $u$ a força (em Newton) aplicada ao carro. Define-se $a=1 /(m+M)$.

De acordo com a metodologia apresentada em (Tanaka and Wang, 2001), o sistema em (29) pode ser descrito por um modelo linear a parâmetros variantes, denominado de modelo fuzzy Takagi-Sugeno. A região de validade $\mathcal{V}_{0}$ considerada para o modelo é definida pelos intervalos $x_{1} \in\left[-x_{1 \max }, x_{1 \max }\right]$ e $x_{2} \in\left[-x_{2 \max }, x_{2 \max }\right]$, sendo para este caso $x_{1 \max }=\pi / 3$ e $x_{2 \max }=5$. Desta forma, tem-se

$$
\begin{gathered}
\dot{x}=\sum_{j=1}^{2} \sum_{k=1}^{2} \sum_{l=1}^{2} \sum_{m=1}^{2} E_{j} M_{k} N_{l} S_{m}\left\{\tilde{A}_{j k l m} x+\tilde{B}_{j k l m} u\right\} \\
\operatorname{com} \tilde{A}_{j k l m}=\left[\begin{array}{cc}
0 & 1 \\
g q_{j} b_{k} & -\frac{a m l}{2} q_{j} c_{l}
\end{array}\right] \text { e } \tilde{B}_{j k l m}=\left[\begin{array}{c}
0 \\
-a q_{j} d_{m}
\end{array}\right] .
\end{gathered}
$$
Então, agregando os somatórios temos

$$
\dot{x}=\sum_{i=1}^{16} h_{i}\left\{\tilde{A}_{i} x+\tilde{B}_{i} u\right\}
$$

sendo $i=m+2(l-1)+4(k-1)+8(j-1) \mathrm{e}$ $h_{i}=E_{j} M_{k} N_{l} S_{m}$ com $E_{1}=\frac{z_{1}-q_{2}}{q_{1}-q_{2}}, E_{2}=\frac{q_{1}-z_{1}}{q_{1}-q_{2}}$, $M_{1}=\frac{\operatorname{sen}\left(x_{1}\right)-\left(\frac{2}{\pi}\right) z_{2}}{\left(1-\frac{2}{\pi}\right) z_{2}}, M_{2}=\frac{x_{1}-z_{2}}{\left(1-\frac{2}{\pi}\right) z_{2}}, N_{1}=\frac{z_{3}-c_{2}}{c_{1}-c_{2}}$, $N_{2}=\frac{c_{1}-z_{3}}{c_{1}-c_{2}}, \quad S_{1}=\frac{z_{4}-d_{2}}{d_{1}-d_{2}}, \quad S_{2}=\frac{d_{1}-z_{4}}{d_{1}-d_{2}}$, $z_{1}=\frac{1}{\frac{4 l}{3}-\operatorname{amlcos}^{2}\left(x_{1}\right)}, z_{2}=\operatorname{sen}\left(x_{1}\right), z_{3}=x_{2} \operatorname{sen}\left(2 x_{1}\right)$, $z_{4}=\cos \left(x_{1}\right)$ e $q_{1}=\max \left(z_{1}\right), q_{2}=\min \left(z_{1}\right), b_{1}=1$, $b_{2}=\frac{2}{\pi}, c_{1}=\max \left(z_{3}\right), c_{2}=\min \left(z_{3}\right), d_{1}=\max \left(z_{4}\right)$, $d_{2}=\min \left(z_{4}\right)$,

nas quais os valores máximos e mínimos são calculados para a região de validade do modelo.

É interessante ressaltar que as funções $h_{i}$ dependem apenas da varíavel $x_{1}$, que é definida como a saída do sis- 
tema. Desta forma, é suficiente a medição da saída para calcular a lei de controle.

\subsection{Exemplo Numérico}

Para o exemplo elege-se: $m=2.0 \mathrm{~kg}, M=8.00 \mathrm{~kg}$ e $2 l=1.0 \mathrm{~m}$. Discretiza-se cada regra em (30) para um tempo de amostragem fixo $T_{s}=0.1 \mathrm{~s}$, com a utilização das seguintes relações (Li et al., 2001)

$$
A_{i}=e^{\tilde{A}_{i} T_{s}}, \quad B_{i}=\int_{0}^{T_{s}} e^{\tilde{A}_{i} \tau} \tilde{B}_{i} d \tau
$$

Desta forma, obtém-se as matrizes que compõem o sistema (1), dispostas no Anexo deste artigo. É importante observar que o modelo em tempo discreto obtido é uma aproximação do modelo fuzzy T-S, devido à variação dos parâmetros ao longo do tempo. Portanto, a escolha do período de amostragem é mais crítica do que no caso de sistemas lineares e invariantes no tempo. Neste trabalho, consideramos uma escolha de $T_{s}$, suficientemente pequeno, e verificamos via simulações que os resultados obtidos garantem o funcionamento seguro, assintoticamente estável, do sistema amostrado em malha-fechada para as condições iniciais pertencentes ao domínio de estabilidade a ser calculado, $\mathcal{L}_{V} \subset \mathcal{X}_{0}$. Cabe salientar que todas as simulações devem ser realizadas considerando a utilização do modelo em tempo discreto do CDRS-PDP ou TDP, com os valores das funções de pertinência calculadas à cada instante de amostragem, e o modelo não linear em tempo contínuo do sistema a controlar.

Como o modelo apresentado é válido localmente, utilizam-se das condições apresentadas nos algoritmos (27) e (28) para síntese dos CDRSs, com

$$
\begin{gathered}
v_{\tau}=\left\{\left[\begin{array}{l}
x_{1 \max } \\
x_{2 \max }
\end{array}\right],\left[\begin{array}{c}
x_{1 \max } \\
-x_{2 \max }
\end{array}\right],\left[\begin{array}{c}
-x_{1 \max } \\
x_{2 \max }
\end{array}\right],\left[\begin{array}{l}
-x_{1 \max } \\
-x_{2 \max }
\end{array}\right]\right\}, \\
\varrho=\left[\begin{array}{c}
x_{1 \max } \\
x_{2 \max }
\end{array}\right] \text { e } L=I_{2} .
\end{gathered}
$$

Na tabela 1 observam-se os valores mínimos do coeficiente de contratividade para os quais os algoritmos (27) e (28) permanecem factíveis. Nota-se que para o CDRSTDP (algoritmo (28)) obtém-se um menor valor do fator de contratividade, possibilitando uma faixa maior para o ajuste de desempenho.

Tabela 1: Factibilidade dos Algoritmos de Controle.

\begin{tabular}{|c|c|}
\hline CDRS - Algoritmo & Fator $\lambda$ \\
\hline PDP (27) & 0,494 \\
\hline TDP $(28)$ & 0,226 \\
\hline
\end{tabular}

Considere a região de estabilidade (RE), definida por:

$$
\begin{gathered}
S_{0 r} \triangleq \bigcap_{i}\left\{\mathscr{E}_{r}\left(\mathbb{Q}_{i}^{-1}\right)\right\} \text { em que } \\
\mathscr{E}_{r}\left(\mathbb{Q}_{i}^{-1}\right)=\left\{x \in \Re^{2} ;\left[\begin{array}{l}
x \\
0
\end{array}\right]^{\prime} \mathbb{Q}_{i}^{-1}\left[\begin{array}{l}
x \\
0
\end{array}\right] \leq 1\right\} .
\end{gathered}
$$

A RE define o conjunto de condições iniciais $\varsigma_{0}=$ $\left[\begin{array}{ll}x_{0}^{\prime} & 0\end{array}\right]^{\prime}$, para as quais as trajetórias do sistema ficam limitadas na região de confinamento $(\mathrm{RC})$. A região $\mathrm{RC}$ é a projeção ortogonal de $\mathscr{E}\left(\mathbb{Q}_{i}^{-1}\right)$ sobre o hiperplano formado pelos estados da planta, definida como

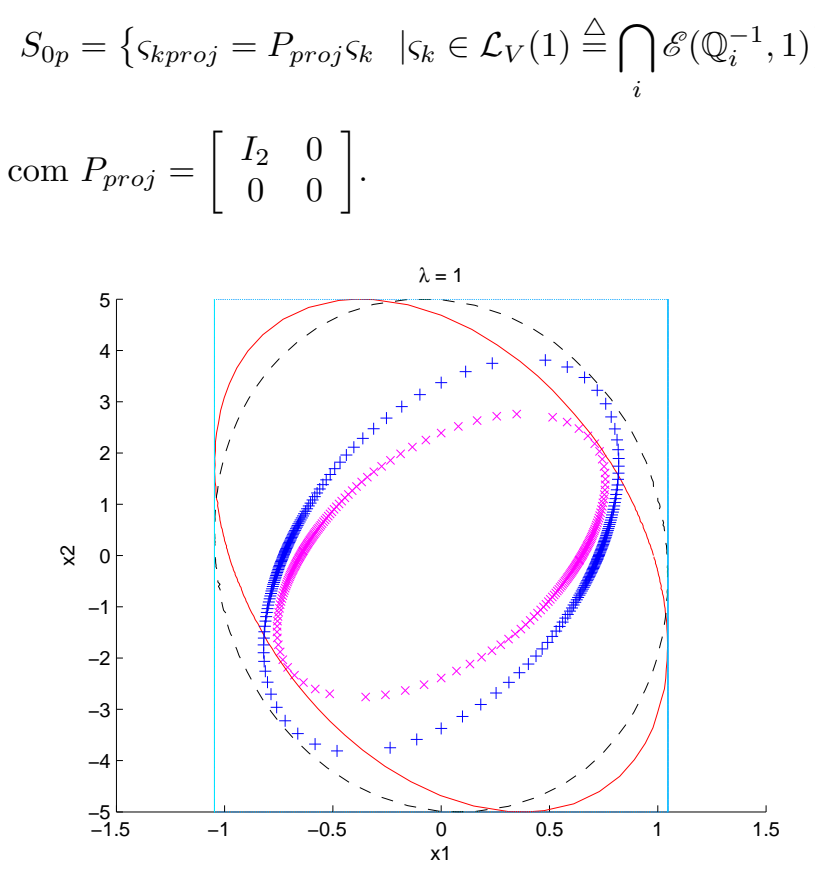

Figura 1: Regiões de Estabilização e Confinamento para $\lambda=1$.

Nas figuras $1(\lambda=1)$ e $2(\lambda=0.7)$ observam-se as regiões de estabilidade (RE), representadas por " $\mathrm{x}$ ", para o CDRS-PDP (CASO 1), e "+", para o CDRS-TDP (CASO 2), e as regiões de confinamento das trajetórias (RC) no espaço de estados da planta, representadas por uma linha contínua para o CASO 1, e uma linha tracejada para o CASO 2.

Nota-se que a exigência de um maior desempenho (menor fator contrativo) provoca uma diminuição das regiões RE e RC, ou seja, observa-se o compromisso existente entre desempempenho e tamanho das regiões de estabilidade e confinamento. Tal fator deve ser considerado pelo projetista. Verifica-se também um menor conservadorismo em termos de tamanho das regiões $\mathrm{RE}$ e RC para o CDRS-TDP (algoritmo (28)), devido ao maior número de graus de liberdade presente na estrutura deste compensador.. 


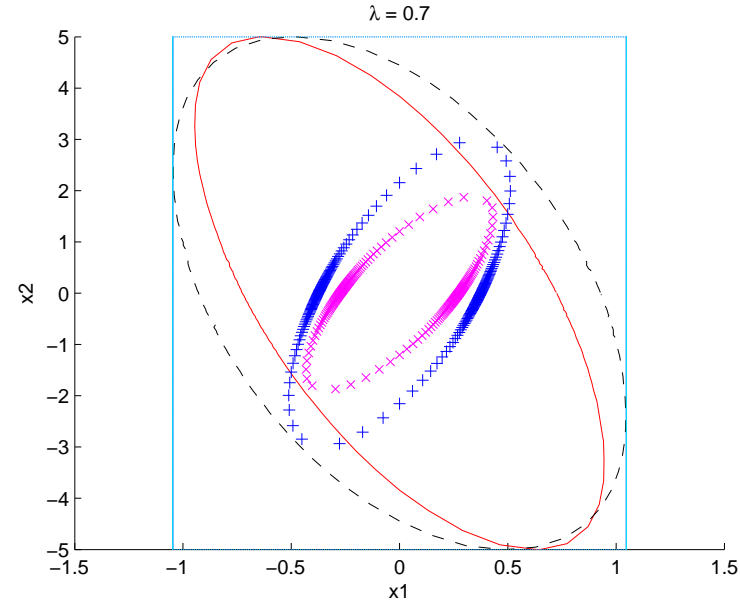

Figura 2: Regiões de Estabilização e Confinamento para $\lambda=$ 0.7 .

Para simulação da evolução temporal dos estados e do esforço de controle, são consideradas as condições iniciais $\varsigma_{0}^{\prime}=\left[\begin{array}{ll}x_{0}^{\prime} & 0\end{array}\right], \operatorname{com} x_{0}^{\prime}=\left[\begin{array}{ll}0.7293 & 2\end{array}\right] \mathrm{e}$ $x_{0}^{\prime}=\left[\begin{array}{ll}0.7569 & 3\end{array}\right]$, para o CDRS-PDP e o CDRSTDP, respectivamente. Nota-se que as condições são escolhidas na fronteira das respectivas regiões de estabilidade.

Nas figuras 3 e 4 observa-se a evolução temporal dos estados da planta para $\lambda=1$, sendo que "+" representa os estados para o CDRS-PDP e "o" para o CDRS-TDP. Nota-se que os estados, para todo instante de tempo, permanecem dentro da região de validade $\mathcal{V}_{0}$. Na figura 5 observam-se os esforços de controle para a mesma condição de simulação anterior.

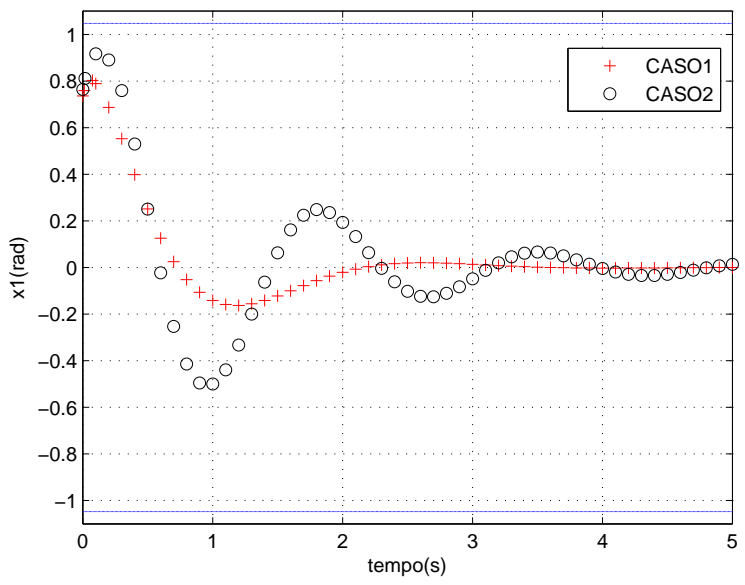

Figura 3: Evolução Temporal de $x_{1}$ para $\lambda=1$.

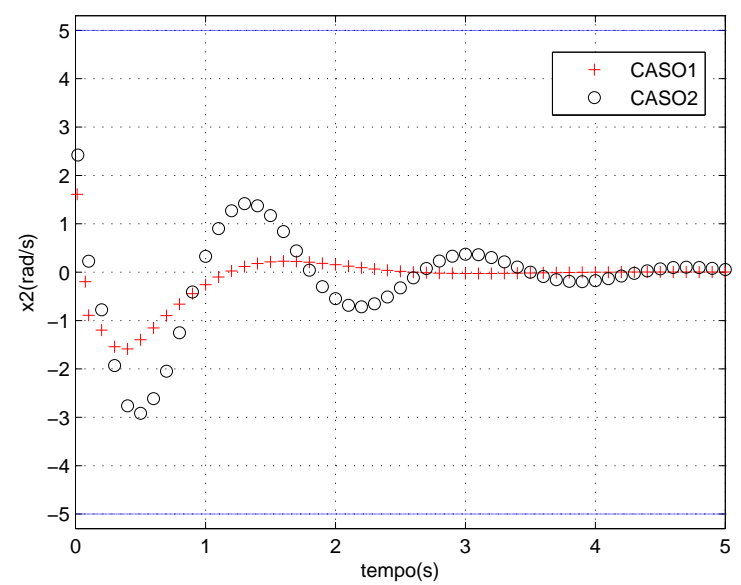

Figura 4: Evolução Temporal de $x_{2}$ para $\lambda=1$.

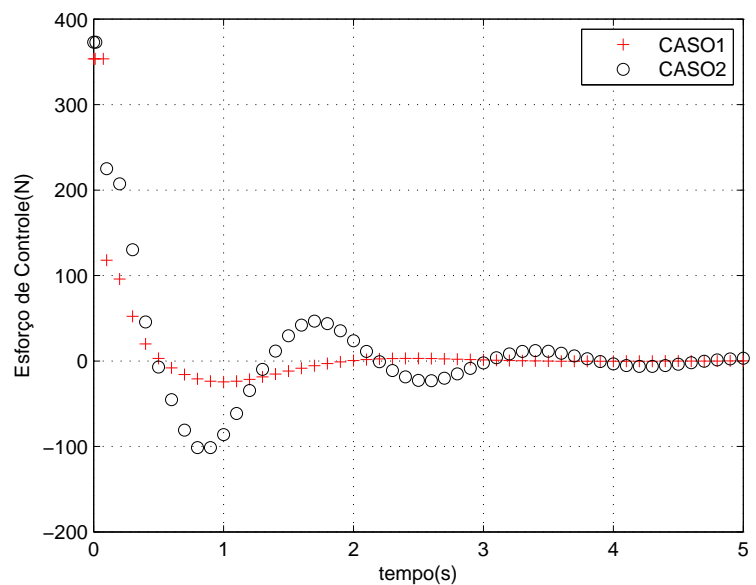

Figura 5: Esforço de Controle para $\lambda=1$.

Por fim, deve-se salientar que para outras condições iniciais não pertencentes à $\mathrm{RE}$ sob consideração, a convergência das trajetórias para a origem não é garantida, mas poderá ocorrer nos casos particulares em que a condição inicial pertencer a região de atração para a origem do sistema não linear em malha-fechada; por exemplo, pode-se verificar via simulação, para o CDRSTDP com coeficiente $\lambda=1$, que as condições iniciais $\varsigma_{0}^{\prime}=\left[\begin{array}{ll}x_{0}^{\prime} & 0\end{array}\right] \notin L_{v}, \operatorname{com} x_{0}^{\prime}=\left[\begin{array}{ll}0.7776 & -1.6228\end{array}\right]$ e $x_{0}^{\prime}=\left[\begin{array}{ll}0 & 3.8158\end{array}\right]$, pertencem à região de atração, e que as condições iniciais com $x_{0}^{\prime}=\left[\begin{array}{ll}0.7085 & -3.7573\end{array}\right]$ e $x_{0}^{\prime}=\left[\begin{array}{ll}0.1279 & 4.8392\end{array}\right]$ implicam em trajetórias instáveis. 


\section{CONCLUSÃO}

Neste artigo foram apresentadas duas estruturas de compensadores dinâmicos por realimentação de saída para o controle de sistemas LPV. Nas estruturas, considera-se a dependência de parâmetros na forma parcial e total.

Para a obtenção de estabilidade e um certo desempenho temporal, utilizou-se de funções de Lyapunov dependente de parâmetros e metodologias adaptadas de (Corso et al., 2009), (Daafouz and Bernussou, 2001) e (Scherer et al., 1997), obtendo-se condições sob a forma de LMI's, e portanto, de solução numérica confiável.

Um aspecto prático de importância discutido no presente artigo está relacionado ao domínio de validade do modelo com parâmetros variantes considerado. Mostrou-se, então, como restrições adicionais podem ser acrescentadas na fase de projeto para garantir a estabilidade local e a evolução das trajetórias do sistema em malha fechada no interior da região de validade do modelo utilizado. Cabe salientar que alguns conceitos utilizados para tratar este aspecto, como a contratividade de conjuntos em relação as trajetórias do sistema em malha fechada e a inclusão de conjuntos, são ferramentas conhecidas e utilizadas, particularmente, na área de controle de sistemas sob restrições (veja (Castelan et al., 2010; Corso et al., 2009) e suas referências).

Finalmente, um exemplo numérico aplicado a um sistema não linear modelado pela técnica fuzzy TakagiSugeno foi exposto, permitindo mostrar alguns aspectos das estratégias de síntese propostas, tais como a relevante consideração prática do domínio de validade mencionado e o compromisso existente entre a exigência de desempenho temporal e o tamanho das regiões de estabilidade e de confinamento para o sistema em malhafechada. Com vistas à uma escolha adequada do período de amostragem e à garantia de estabilidade do sistema de controle, um ponto importante para investigação futura é a análise da região de estabilidade, calculada a partir do modelo discretizado de sistemas LPV, quando da implementação sobre o sistema em tempo contínuo.

\section{AGRADECIMENTOS}

Os autores agradecem ao Editor e aos Revisores pelos comentários e sugestões realizados, e à CAPES e ao CNPq pelo suporte financeiro.

\section{ANEXO}

Matrizes que compõem a estrutura do sistema para o exemplo numérico:

$$
\begin{aligned}
& A_{1}=\left[\begin{array}{ll}
1.0864 & 0.1007 \\
1.7410 & 1.0420
\end{array}\right], \quad B_{1}=\left[\begin{array}{l}
-0.0009 \\
-0.0178
\end{array}\right], \\
& A_{2}=\left[\begin{array}{ll}
1.0864 & 0.1007 \\
1.7410 & 1.0420
\end{array}\right], \quad B_{2}=\left[\begin{array}{l}
-0.0004 \\
-0.0089
\end{array}\right], \\
& A_{3}=\left[\begin{array}{ll}
1.0890 & 0.1052 \\
1.8195 & 1.1354
\end{array}\right], \quad B_{3}=\left[\begin{array}{l}
-0.0009 \\
-0.0186
\end{array}\right], \\
& A_{4}=\left[\begin{array}{ll}
1.0890 & 0.1052 \\
1.8195 & 1.1354
\end{array}\right], \quad B_{4}=\left[\begin{array}{l}
-0.0005 \\
-0.0093
\end{array}\right], \\
& A_{5}=\left[\begin{array}{ll}
1.0547 & 0.0996 \\
1.0969 & 1.0108
\end{array}\right], \quad B_{5}=\left[\begin{array}{l}
-0.0009 \\
-0.0176
\end{array}\right], \\
& A_{6}=\left[\begin{array}{ll}
1.0547 & 0.0996 \\
1.0969 & 1.0108
\end{array}\right], \quad B_{6}=\left[\begin{array}{l}
-0.0004 \\
-0.0088
\end{array}\right], \\
& A_{7}=\left[\begin{array}{ll}
1.0564 & 0.1041 \\
1.1464 & 1.1023
\end{array}\right], \quad B_{7}=\left[\begin{array}{l}
-0.0009 \\
-0.0184
\end{array}\right], \\
& A_{8}=\left[\begin{array}{ll}
1.0564 & 0.1041 \\
1.1464 & 1.1023
\end{array}\right], \quad B_{8}=\left[\begin{array}{l}
-0.0005 \\
-0.0092
\end{array}\right], \\
& A_{9}=\left[\begin{array}{ll}
1.0763 & 0.1006 \\
1.5363 & 1.0372
\end{array}\right], \quad B_{9}=\left[\begin{array}{l}
-0.0008 \\
-0.0157
\end{array}\right], \\
& A_{10}=\left[\begin{array}{ll}
1.0763 & 0.1006 \\
1.5363 & 1.0372
\end{array}\right], \quad B_{10}=\left[\begin{array}{l}
-0.0004 \\
-0.0078
\end{array}\right], \\
& A_{11}=\left[\begin{array}{ll}
1.0784 & 0.1046 \\
1.5974 & 1.1191
\end{array}\right], \quad B_{11}=\left[\begin{array}{l}
-0.0008 \\
-0.0163
\end{array}\right], \\
& A_{12}=\left[\begin{array}{ll}
1.0784 & 0.1046 \\
1.5974 & 1.1191
\end{array}\right], \quad B_{12}=\left[\begin{array}{l}
-0.0004 \\
-0.0081
\end{array}\right], \\
& A_{13}=\left[\begin{array}{ll}
1.0484 & 0.0997 \\
0.9691 & 1.0095
\end{array}\right], \quad B_{13}=\left[\begin{array}{l}
-0.0008 \\
-0.0155
\end{array}\right], \\
& A_{14}=\left[\begin{array}{ll}
1.0484 & 0.0997 \\
0.9691 & 1.0095
\end{array}\right], \quad B_{14}=\left[\begin{array}{l}
-0.0004 \\
-0.0078
\end{array}\right], \\
& A_{15}=\left[\begin{array}{ll}
1.0497 & 0.1036 \\
1.0076 & 1.0900
\end{array}\right], \quad B_{15}=\left[\begin{array}{l}
-0.0008 \\
-0.0162
\end{array}\right], \\
& A_{16}=\left[\begin{array}{ll}
1.0497 & 0.1036 \\
1.0076 & 1.0900
\end{array}\right], \quad B_{16}=\left[\begin{array}{l}
-0.0004 \\
-0.0081
\end{array}\right]
\end{aligned}
$$

\section{REFERÊNCIAS}

Andrea, C. Q., Pinto, J. O. P., Assunção, E., Teixeira, M. C. M. and Junior, L. G. (2008). Controle ótimo $H_{\infty}$ de sistemas não lineares com modelos fuzzy Takagi-Sugeno, Revista Controle \& Automação 19(3):256-269.

Araújo, L. O., Pellanda, P. C. and Galdino, J. F. (2010). Técnica de representação de modelos LPV baseada na transformada Haar, Congresso Brasileiro de Automática, Bonito-MS, Brazil, pp.3197-3204.

Arzen, K. E. and Cervin, A. (2005). Control and embedded computing: survey of research direction, Proc. of 16th IFAC World Congress, Prague, Czech Republic.

Boyd, S., Ghaoui, L., Feron, E. and Balakrishnan, V. (1994). Linear matrix inequalities in system and control Theory, SIAM Studies in Applied Mathematics, 1994.

Cannon, R. H. (1967). Dynamics of physical systems, McGrawHill, New York. 
Castelan, E. B., Leite, V. J. S., Miranda, M. F. and Moraes, V. M. (2010). Syntesis of output feedback controllers for a class of nonlinear parameter-varying discrete-time systems subject to actuators limitations, Proc. of American Control Conference, Baltimore, USA, pp.4235-4240.

Corso, J., Castelan, E. B., Moreno, U. F. and Pieri, E. R. (2009). Controle dependente de parâmetros para uma classe de sistemas não lineares incertos com atuadores saturantes, Revista Controle \& Automação 20(2):119-132.

Daafouz, J. and Bernussou, J. (2001). Parameter dependent lyapunov functions for discrete time systems with time varying parametric uncertainties, Systems \& Control Letters 43(5):355-359.

Geromel, J. C. and Colaneri, P. (2006). Robust stability of time varying polytopic systems, Systems \& Control Letters $\mathbf{5 5}(1): 81-85$.

Guerra, T. M. and Vermeiren, L. (2004). LMI-based relaxed nonquadratic stabilization conditions for nonlinear systems in the Takagi-Sugeno's form, Automatica 40(5):823-829.

$\mathrm{Hu}, \mathrm{T}$., Lin Z. and Chen B. M. (2002). An analysis and design method for linear systems subject to actuator saturation and disturbance, Automatica 38(2):351-359.

Khiar, D., Lauber, J., Floquet, T., Colin, G., Guerra, T. M. and Chamaillard, Y. (2007). Robust Takagi-Sugeno fuzzy control of a spark ignition engine, Control Engineering Practice 15(12):1446-1456.

Klug, M., Castelan, E. B. and Leite, V. J. S. (2011). A dynamic compensator for parameter varying systems subject to actuator limitations applied to a T-S fuzzy system, Proc. of 18th IFAC World Congress, Milan, Italy, pp.14495-14500.

Leite, V. J. S., Montagner, V. F., Oliveira, P. J., Oliveira, R. C. K. F., Ramos, D. C. W. and Peres, P. L. D. (2004). Estabilidade robusta de sistemas lineares através de desigualdades matriciais lineares, Revista Controle \& Automação 15(1):24-40.

Li, Z., Park, J. B. and Joo, Y. H. (2001). Chaotifying continuoustime TS fuzzy systems via discretization, IEEE Transactions on Circuits and Systems - I: Fundamental Theory and Applications 48(10):1237-1243.

Milani, B. E. A. and Coelho, A. D. (2002). Funções de Lyapunov Lineares por Partes para Sistemas Lineares com Controles Saturáveis, Revista Controle \& Automação 13(1):42-50.

Montagner, V. F., Oliveira, R. C. L. F., Leite, V. J. S. and Peres, P. L. D. (2005). LMI approach for $H_{\infty}$ linear parametervarying state feedback control, IEEE Proceedings in Control Theory Applications 152(2):195-201.

Montagner, V. F., Oliveira, R. C. and Peres, P. L. D. (2010). Relaxações convexas de convergência garantida para o projeto de controladores para sistemas nebulosos de Takagi Sugeno, Revista Controle \& Automação 21(1):82-95.

Mozelli, L. A. and Palhares, R. M. (2011). Stability Analysis of Linear Time-Varying Systems: Improving Conditions by Adding More Information About Parameter Variation, Systems \& Control Letters 60(5):338-343.

Oliveira, J., Trofino, A. and Souza, C. E. (2002). Análise e síntese $H_{\infty}$ para sistemas $L P V$, Revista Controle \& Automação 13(1):6-12

Oliveira, R. C. L. F. and Peres, P. L. D. (2008). Robust stability analysis and control design for time-varying discrete-time polytopic systems with bounded parameter variation, Proc. of American Control Conference, Seattle, USA, pp.3094-3099.
Scherer, C., Gahinet, P. and Chilali, M. (1997). Multiobjective output-feedback control via LMI optimization, IEEE Transactions on Automatic Control 42(7):896-911.

Souza, C. E. and Trofino, A. (2005). Gain-sheduled $\mathrm{H}_{2}$ controller synthesis for linear parameter varying systems via parameter-dependent Lyapunov functions, International Journal of Robust and Nonlinear Control 16(5):243-257.

Tanaka, K. and Wang, H. O. (2001). Fuzzy control systems design and analysis: a linear matrix inequality approach, John Wiley \& Sons, New York.

Tognetti, E. S., Oliveira, R. C. L. F. and Peres, P. L. D. (2009). LMI relaxations for nonquadratic stabilization of discretetime Takagi-Sugeno systems based on polynomial fuzzy Lyapunov functions, Proc. of 17th Mediterranean Conference on Control and Automation, Thessaloniki, Greece, pp.7-12.

Trofino, A. and Souza, C. E. (2001). Biquadratic stability of uncertain linear systems, IEEE Transactions on Automatic Control 46(8):1303-1307.

Wang, H. O., Tanaka, K. and Griffin, M. F. (1996). An approach to fuzzy control of nonlinear systems: stability and design issues, IEEE Transactions on Fuzzy Systems 4(1):14-23.

Zheng, F., Wang, Q., Lee, T. H. and Huang, X. (2001). Robust PI controller design for nonlinar systems via fuzzy modeling approach, IEEE Transactions on Systems, Man, and Cybernetics 31(6):666-675.

Zhou, S., Lam, J. and Zheng, W. X. (2007). Control design for fuzzy systems based on relaxed nonquadratic stability and $H_{\infty}$ performance conditions, IEEE Transactions on Fuzzy Systems 15(2):188-199. 